\title{
ENHANCED PLATFORM FOR BUILDING INFORMATION MODELING TO IMPROVE REALITY
}

\author{
Boong Yeol Ryoo $^{1} *$ and Hyung Keun Park ${ }^{2}$ \\ ${ }^{1}$ Department of Construction Science, Texas A\&M University, Texas, USA. \\ ${ }^{2}$ School of Civil Engineering, Chungbuk National University, Cheongju, Korea. \\ *Corresponding author (bryoo@tamu.edu)
}

\begin{abstract}
In order to improve the mobility and practicality of Building Information Models (BIM), a framework using a multimedia device, a global positioning system (GPS) and inclinometer is proposed. The GPS receiver provides location and an inclinometer measures angle of slope, both inclines and declines, of an observer. Based on these two additional inputs, the proposed system displays a BIM element on the real world picture as seen by an observer looking upward or downward. It would improve the practicality of BIM models during the planning phase, the mobility of the model during the construction, and operations, throughout maintenance phases.
\end{abstract}

Keywords: Mixed Reality, Building Information Model (BIM), Global Positioning System (GPS), Inclinometer, Augmented Reality (AR)

\section{INTRODUCTION}

Building Information Model (BIM) has been an emerging technology in the construction industry along with Virtual Reality (VR) [1]. Construction contractors focus on 3D/4D visualization and constructability analysis with mainly clash detection during the construction phase. McGraw Hill's BIM executive summary shows that $87 \%$ of BIM expert users are experiencing positive returns on investment with BIM, and 93\% of BIM users believe there is potential to gain more value from BIM in the future [2]. Various efforts have been taken to improve the applicability of BIM but a lack of effort to improve the mobility of BIM has been noticed [3]. Augmented Reality (AR) is a promising tool in mixed reality but it has not been fully implemented in the construction industry. Regardless of their efforts, contractors are struggling with the same barriers as were common during the initial implementation of BIM. Due to a limited mobility, BIM has not been fully implemented as an Augmented Reality (AR) tool. Van Krevelen and Poelmane introduced a potential application which can be used in the construction industry such as pedestrian navigation and traffic warning AR [4]
This paper focuses on integrating BIM with wireless/mobile sensors to improve the mobility and practicality of BIM models.

\section{OBJECTIVES}

This research is to propose a framework for real-time interactive BIM models as a real-time interactive visualization tool. The framework is to present a wireframe BIM model on the real world pictures using GPS coordinates and observation angles, either inclines or declines, as seen by an observer. Thus BIM is linked to the real world. This enables an observer to perform 3D real time/world walk-through as the observer actually navigates the proposed building site. This research aims at validating:

- Technical configurations of the proposed system

- usability of inclinometer which is a pitch and roll indicator

- a mathematical model for transforming 3D images using GPS coordinates and inclines or declines

- integration of BIM images and real world images

- information breakdown structure for the framework 


\section{FRAMEWORK AND CONFIGURATION OF REAL-TIME BIM}

The addition of wireless/mobile sensors such as GPS sensors for collecting location, inclinometers for measuring an observer's view direction and angles, and wire-frame BIM models transforms a static BIM to a mobile BIM as shown in Fig. 1.

In order to improve the mobility of BIM, linking BIM models to real world coordinates, the location of an observer, should be collected. The coordinates are then translated into local coordinates in the virtual world. A piece of the BIM model is linked to the real world image through the local coordinates.

A hardhat was equipped with three axis sensors (inclinometers) as well as a camera. The axis sensors relay information to a GPS device which tells where they are viewing and the camera records what is actually being viewed. Based on the direction that an observer faces and the incline or decline angle, an associated element of the BIM model will be selected and displayed. To transform 3D world (BIM elements) into 2D plan (live pictures), a 3D view image of a BIM element was first created, projected, and then mapped onto the specified location on the screen.

An integrated database contains building element data, geometry data, and property data. A space map groups related BIM elements each other such as floor, walls, and ceiling as in the same space/room. This matrix plays a key role in the framework since BIM elements are loosely connected and stored in the database.

In order to transform BIM into AR, a head mounted display (HMD) was used to improve the mobility of the BIM. However a portable PC can substitute the HMD.

3D transformation calculations were performed to update the images of BIM elements as an observer navigates a future project site the framework calculates transformation matrix the image of building

\section{CONCLUSIONS AND FUTURE WORK}

The configuration for the proposed framework proves itself technically feasible. Despite the fact that wire-frame BIM models were used to validate the framework and configurations, the proposed approach promises better mobility and practicability of BIM models. The integrated property databases were essential to keep the framework simple, light, and easy to maintain. It suggests information based BIM modeling can be feasible. Series of full scale field tests are expected to review further technical feasibility of the framework with existing BIM programs.

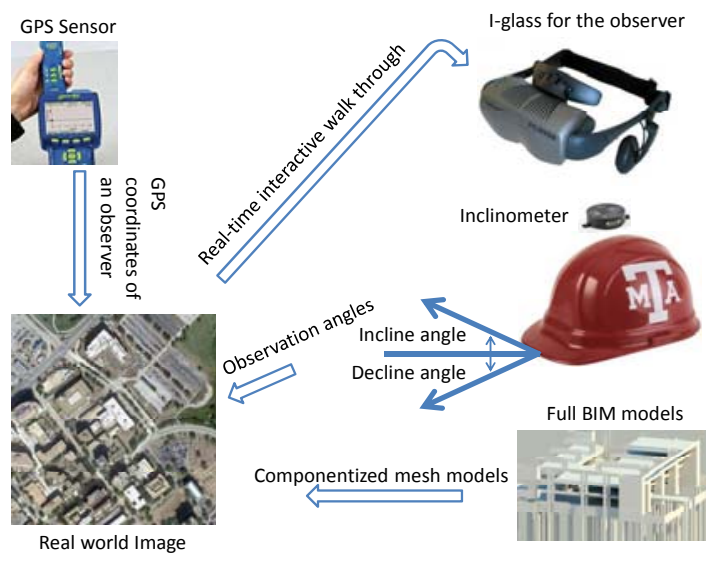

Fig. 1 Framework of the proposed BIM Model

\section{REFERENCES}

[1] Gu, N. and London, K. "Understanding and Facilitating BIM Adoption in the AEC Industry”, Automation in Construction, 19(8), pp. 998-999, 2010.

[2] McGraw Hill, "The Business Value of BIM - Getting Building Information Modeling to the Bottom Line”, McGraw Hill Smart Market Report 2009, McGraw Hill, 2009.

[3] Jung, Y. and Joo, M., “Building Information Modeling (BIM) Framework for Practical Implementation”, Automation in Construction, 20(2), pp. 126-133, March 2011.

[4] van Krevenlen, D.W.F. and Poelman, R., “A Survey of Augmented Reality Technology, Applications and Limitations”, The International Journal of Virtual Reality, 9(2), pp. 1-20, 2010. 